\title{
Unidade de Contexto \\ e Observação Social Sistemática em Saúde: Conceitos e Métodos
}

\author{
I ${ }^{1}$ Fernando Augusto Proietti, ${ }^{2}$ Cláudia Di Lorenzo Oliveira, ${ }^{3}$ Fabiane Ribeiro Ferreira, 4 \\ Aline Dayrell Ferreira, ${ }^{5}$ Waleska Teixeira Caiaffa I
}

Resumo: Assumimos que "onde você mora é importante para sua saúde, para além de quem você é". Entendemos que o impacto do local de moradia ou unidade de contexto (UC) na saúde das populaçôes se deve à heterogeneidade dos atributos do entorno físico e social da UC, para além das características individuais ou agregadas daqueles ali aninhados. Estes atributos, embora dependentes dos indivíduos, são tipicamente externos a eles e potencialmente modificáveis. As UC são compreendidas como unidades ecológicas inseridas em conjuntos sucessivamente mais amplos e interdependentes. Quando relevante para a hipótese do estudo, unidades geográficas administrativas podem ser utilizadas como aproximações da UC. Outra alternativa é a que utiliza a percepção de seus moradores, a "vizinhança percebida". O ressurgimento do interesse com relação à determinação dos efeitos da UC sobre a saúde correlaciona com novas tendências na área da saúde coletiva: incorporação de novos níveis hierárquicos de exposição, as iniquiidades e seus determinantes, a urbanização e seus efeitos e a avaliação de intervençôes multi-setoriais. Nosso objetivo central é rever opçōes para a escolha da UC a ser investigada além de estratégias para a aferição de seus atributos físicos e sociais, utilizando a observação social sistemática (OSS). A combinação de dados originárias de dados administrativos, da vizinhança percebida, dos inquéritos populacionais e da OSS ainda necessita de maiores elaboraçôes conceitual, metodológica e analítica.

Entretanto, a compreensão da distribuição dos atributos físicos e sociais da UC permite compor níveis hierárquicos de complexidade relevantes para o entendimento da ocorrência dos eventos relacionados à saúde nas populaçôes.

\author{
1 Observatório de Saúde \\ Urbana, Universidade Federal \\ de Minas Gerais (UFMG); \\ Grupo de Pesquisa em \\ Epidemiologia (GPE/CNPq); \\ Departamento de Medicina \\ Social e Preventiva. Endereço \\ eletrônico: \\ proietti@medicina.ufmg.br \\ 2 Observatório de Saúde \\ Urbana, Universidade Federal \\ de Minas Gerais (UFMG); \\ Grupo de Pesquisa em \\ Epidemiologia (GPE/CNPq); \\ Fundação Hemominas, Belo \\ Horizonte, Minas Gerais, Brasil. \\ ${ }^{3}$ Observatório de Saúde \\ Urbana, Universidade Federal \\ de Minas Gerais (UFMG); \\ Grupo de Pesquisa em \\ Epidemiologia (GPE/CNPq). \\ ${ }^{4}$ Observatório de Saúde \\ Urbana, Universidade Federal \\ de Minas Gerais (UFMG); \\ Grupo de Pesquisa em \\ Epidemiologia (GPE/CNPq); \\ Departamento Materno- \\ Infantil e Saúde Coletiva, \\ Escola de Enfermagem, \\ Universidade Federal de Minas \\ Gerais (UFMG). \\ ${ }^{5}$ Observatório de Saúde \\ Urbana, Universidade Federal \\ de Minas Gerais (UFMG); \\ Grupo de Pesquisa em \\ Epidemiologia (GPE/CNPq); \\ Departamento de Medicina \\ Social e Preventiva.
}




\section{Introdução}

Nos últimos anos, o renovado interesse na mensuração do impacto dos atributos físicos e sociais da unidade geográfica de moradia sobre a saúde das populações passou a ocupar importante papel na agenda da saúde coletiva e saúde urbana (DIEZ-ROUX, 1998 e 2001). No entanto, há aproximadamente 40 anos, Harburg et al. (1973) avaliaram se vizinhanças caracterizadas como "áreas de estresse elevado” influenciavam a pressão arterial, controlando para determinantes individuais entendidos como fatores de confusão e concluíram que residir nessas áreas estava associado a níveis de pressão arterial sistólica e diastólica aumentados, independentemente dos atributos individuais.

Assumindo que "onde você mora é importante para sua saúde, para além de quem você é” (KAWACHI; BERKMAN, 2003), neste artigo denominamos o local de residência em centros urbanos, com suas características físicas e sociais, como unidade de contexto (UC). Quando relevante para a hipótese sendo investigada, unidades administrativas com limites geográficos previamente delimitados (por exemplo, setor censitário, bairro, área de abrangência de uma unidade básica de saúde) podem ser utilizadas como aproximaçôes da UC. Uma outra alternativa para a definição de uma UC, recentemente relatada em estudos internacionais, é a que utiliza a percepção de seus moradores, aqui denominada "vizinhança percebida". Devemos ressaltar que a UC percebida não necessariamente sobrepõe-se às unidades administrativas. Em ambas alternativas, as UC são compreendidas como unidades ecológicas inseridas em conjuntos sucessivamente mais amplos e interdependentes (SAMPSON et al., 2002).

Entendemos também que a ocorrência dos eventos relacionados à saúde (ERS) resulta da complexa relação entre atributos individuais (nível hierárquico individual), atributos do agregado, grupo ou população em que os indivíduos estão aninhados (nível hierárquico agregado) e do entorno físico e social da UC (contexto).

Oportunidades para a mudança de estilos de vida, como tabagismo, hábitos alimentares e sedentarismo, intimamente associados a um aumento relativo da morbimortalidade e deterioração da qualidade de vida das populações, podem ser criadas ou limitadas nas UC de centros urbanos. O acesso a serviços de saúde e fontes comerciais de alimentação saudável, assim como a serviços e equipamentos urbanos vêm sendo cada vez mais investigados como determinantes de hábitos e comportamentos que vão além das características dos indivíduos. Portanto, a vizinhança pode ser entendida 
como um coletivo amistoso, compondo uma rede local de suporte ou representando ameaça e insegurança. Em outras palavras, a área de moradia pode ser percebida como amigável, local de encontro de velhos amigos ou território a ser evitado (RAUDENBUSH; SAMPSON, 1999; RAUDENBUSH, 2003).

O ressurgimento do interesse no campo da epidemiologia e saúde coletiva com relação à determinação dos efeitos da UC sobre a saúde também se correlaciona com novas tendências na área: a incorporação de novos níveis hierárquicos de exposição, as iniqüidades e seus determinantes, a urbanização e seus efeitos e a avaliação de impacto de intervençōes multi-setoriais.

A primeira dessas tendências reflete o entendimento de que os determinantes mensurados e restritos em nível individual são insuficientes, não capturando relevantes informações sobre a distribuição dos ERS nas populações. Ainda, uma importante contribuição para o crescente interesse em determinar e mensurar o impacto do local de moradia tem sido a renovada compreensão das causas das iniqüidades em saúde (KRIEGER, 1994; SCHWARTZ et al., 1999; BLAKELY; WOODWARD, 2000; DIEZ-ROUX, 2004; BARATA, 2005; SANTOS et al., 2007; OMPAD et al., 2007; CAIAFFA et al., no prelo).

Quanto à urbanização, o viver na cidade é certamente uma relevante mudança demográfica ocorrida nas últimas décadas. As cidades representam metabolismos complexos que, com suas redes e conexões, têm relevantes implicações em saúde, incluindo a exacerbação dos problemas sociais como a violência, os acidentes de trânsito, a presença de doenças emergentes e reemergentes, transmissíveis ou não. Neste mundo contemporâneo, predominantemente urbano, os determinantes sociais de saúde operam por meio de processos diversos dentro das UC. Nesta perspectiva, a epidemiologia e a saúde coletiva devem considerar o impacto na saúde de intervenções multi-setoriais sobre o entorno físico e social, que não são tradicionalmente associadas à área de saúde (MORGAN; CHINN, 1983; JONES; DUNCAN, 1995; O'CAMPO et al., 1995; DIEZ-ROUX et al., 1997; SLOGGETT; JOSHI, 1998; REJNEVEL, 1998; COHEN et al., 2000; BOSMA et al., 2001; DIEZ-ROUX et al., 2001; MORLAND et al., 2002; BALFOUR; KAPLAN, 2002; DIEZ-ROUX, 2003; CAIAFFA et al., 2005, VLAHOV et al., 2007).

Recentemente, alguns estudos avaliaram associaçōes entre características do entorno físico e social da UC e diferentes ERS, como a autopercepção da saúde, comportamentos e estilos de vida, morbidade e mortalidade decorrentes de doenças 
crônico-degenerativas, saúde mental, saúde da criança e eventos associados à gravidez e ao parto. Extensa revisão da literatura publicada por Pickett \& Pearl (2001) objetivou avaliar o impacto das características socioeconômicas da UC na saúde das populações. Entre os 25 estudos revistos por esses autores, 23 relataram associação entre indicadores socioeconômicos da UC e a ocorrência de diversos ERS, considerando simultaneamente a posição socioeconômica individual dos moradores (PICKETT; PEARL, 2001).

O objetivo central deste artigo é discutir conceitualmente possibilidades e opçôes para a escolha da unidade de contexto de moradia a ser investigada além de estratégias para a aferição dos atributos físicos e sociais desta mesma unidade geográfica de residência. A abordagem dos conceitos e métodos foi conduzida utilizando-se publicações específicas, não resultando portanto de revisão exaustiva da literatura.

\section{Mensuração das características da unidade de contexto}

Assumimos neste texto que o impacto das UC na saúde das populações se deve à heterogeneidade dos atributos do entorno físico e social, para além das características individuais ou agregadas daqueles ali aninhados. Esses atributos, embora dependentes dos indivíduos, são tipicamente externos a eles e potencialmente modificáveis.

A mensuração das propriedades do entorno físico e social requer estratégias diversas. No campo da epidemiologia, a mensuração desses atributos dentro de uma UC é menos estudada e sistematizada, e ainda, metodologicamente menos sofisticada e desenvolvida quando comparada à mensuração das características dos indivíduos (por exemplo, biológicas e estilos de vida).

Os modelos prevalentes e dominantes no campo da epidemiologia procuram explicar a heterogeneidade dos ERS, ocorrendo nos indivíduos e populaçóes como resultante de determinantes medidos em nível individual. Subjacente a esta aparente simplificação, está a premissa ou modelo conceitual de que os determinantes dos ERS são mais bem conceituados e medidos neste nível. Ainda de acordo com este modelo, atributos físicos e sociais, enquanto propriedades do agregado e contexto, são somente indicadores dos determinantes individuais, quando estes não estão disponíveis ou não podem ser medidos. Populações ou grupos e suas UC específicas são considerados apenas coleções de unidades individuais independentes (mesmo quando da análise estatística) e não como entidades per si, com propriedades 
próprias que podem impactar as unidades individuais que as compõem (SUSSER; SUSSER, 1996a e 1996b; SCHWARTZ et al., 1999).

Para uma melhor compreensão dos itens que se seguem, algumas definições de termos e conceitos são necessárias ao se investigar associações entre os atributos das UC e ERS. Como mencionamos acima, três níveis hierárquicos ou de complexidade são possíveis: individual, agregado e contextual. Variáveis que capturam características ou construtos dos indivíduos (por exemplo, anos de estudo) são denominadas individuais; aquelas que refletem propriedades do agregado, tendo contrapartida em nível individual (por exemplo, a renda per capita média da UC) são descritas como variáveis de composição. As variáveis que caracterizam os atributos físicos e sociais da UC, não resultando da agregação de características dos indivíduos nela aninhados, referimos como integrais. Como exemplo de variável integral, Cohen et al. (2000) investigaram a associação entre incidência de gonorréia e características físicas do local de moradia, utilizando o "índice de janelas quebradas" como marcador da deterioração física da UC. Este índice foi composto por três variáveis integrais, entre elas a "percentagem de ruas da UC com lixo, carros abandonados e grafite", obtida por observação direta.

\section{Delimitação da Unidade de Contexto}

A definição da UC, relevante para a hipótese em estudo, representa uma das questôes centrais para assegurar a qualidade da investigação. Como mencionamos anteriormente, duas estratégias têm sido reportadas na literatura: a utilização de unidades administrativas e a "vizinhança percebida".

\section{UC de origem administrativa}

A estratégia mais frequentemente citada na literatura para a definição de uma UC é sua delimitação referenciada em unidades administrativas, com limites geográficos previamente delimitados (PICKETT; PEARL, 2001; WERNECK et al., 2007; ALMEIDA et al., 2007). A principal vantagem desta metodologia é a possibilidade da utilização de informações secundárias já disponíveis em diversas bases de dados. Quando associada a inquéritos populacionais, possibilita uma visão mais integrada da UC.

Uma outra alternativa é a delimitação de áreas a partir de informaçōes geográficas obtidas com imagens de satélite, fotos aéreas e/ou informações 
georreferenciadas (GIS) (MOORE et al., 2008). Esta estratégia pode ser uma opção para garantir homogeneidade física maior do que a opção anterior, ao considerar barreiras geográficas e áreas de ocupação muito diferenciadas, resultando em especificação mais adequada da UC.

A utilização das unidades administrativas, como aproximação para a UC, tem como limitaçóes a incerteza quanto à homogeneidade física e social das áreas e sua possível não-representação e correspondência com a vizinhança enquanto relevante para a população de estudo. Adicionalmente, tais unidades podem ser inapropriadas para a hipótese em estudo, tanto em relação à distribuição geográfica dos determinantes de interesse quanto aos ERS, podendo resultar em viés de informação, dentre outros (PICKETT; PEARL, 2001; CAUGHY et al., 2001; DIEZ-ROUX, 2007).

\section{UCpercebida}

A UC muitas vezes não necessariamente corresponde à delimitação geográfica ou administrativa, podendo ir além ou aquém da mesma. Se considerarmos a UC como um ambiente onde as pessoas vivem e desenvolvem suas atividades diárias, a mesma pode ser aferida a partir da percepção dos indivíduos nela aninhados ( $\mathrm{CHO}$ et al., 2005; WALKER; HILLER, 2007).

Várias metodologias para aferição da percepção do ambiente têm sido utilizadas englobando estudos qualitativos com entrevistas em profundidade (WALKER; HILLER, 2007) e quantitativos, usando instrumentos predefinidos (BRENNAN et al., 2003; BOSLAUGH et al., 2004; CHO et al., 2005). No entanto, nota-se uma deficiência no desenvolvimento, validação e padronização dos instrumentos dificultando aferição e comparabilidade, principalmente em estudos epidemiológicos (MUJAHID et al., 2007).

O desenvolvimento e validação de uma escala do tipo Likert, denominada Neighbourhood Quality Index, para medir a satisfação com a vizinhança, em um estudo de base populacional, destaca-se como um dos instrumentos contemporâneos nesta área. A escala proposta contém 15 itens distribuídos em três domínios: percepção do capital social, percepção da segurança e adequação dos serviços e equipamentos (YANG et al., 2002).

Ressalta-se que, com a transição demográfica e epidemiológica, estudos mais específicos que utilizam a percepção do macroambiente da UC têm sido freqüentemente realizados com o objetivo de relacionar os fatores facilitadores e 
dificultadores do ambiente associados às doenças. Destacam-se, dentre outros, os estudos internacionais sobre capital social (HARPHAM et al., 2002; SILVA et al., 2006) e fatores de risco para doenças cardiovasculares e práticas de atividade física (BRENNAN et al., 2003; BOSLAUGH et al., 2004; MOORE; DIEZ-ROUX, 2006; MUJAHID et al., 2007; NIELSEN; HANSEN, 2007; DIEZ-ROUX, 2007; AUCHINCLOSS et al., 2007). No Brasil, o Inquérito de Saúde dos Adultos na Região Metropolitana de Belo Horizonte avaliou a percepção do entorno social da vizinhança e morbidade auto-referida (LIMA-COSTA, 2004).

Resultante desse movimento, instrumentos podem hoje ser encontrados na literatura. Destacam-se, neste sentido, os estudos do MESA (Multi-Ethnic Study of Atherosclerosis), tendo como objeto de investigação as doenças cardiovasculares (BILD et al., 2002) e o estudo publicado por Brownson et al. (2004), comparando três instrumentos previamente testados quanto a suas propriedades psicométricas, capazes de avaliar o ambiente físico e social: San Diego Instrument, South Carolina Instrument e St Louis Instrument.

Observa-se uma tendência de valorização de instrumentos nesta área, contendo características híbridas de aferição. Objetivam captar a percepção tanto do ambiente físico como do social de populações, principalmente em centros urbanos. Neste tipo de metodologia, a definição da UC tanto pode utilizar mapas da área e solicitar que os residentes identifiquem seus limites de vizinhança, quanto estabelecer medidas objetivas, tais como número de quarteirões ou tempo de caminhada para ir de um ponto ao outro da vizinhança (MOORE et al., 2008). A maior desvantagem é que em geral não é possível uma padronização, e a comparação entre estudos e/ou a incorporação de informações de outras bases de dados torna-se difícil.

A possibilidade da ocorrência do viés de fonte comum de informação (same source bias) deve ser também considerada. Este viés ocorre quando o participante informa simultaneamente o ERS de interesse e os atributos da UC, resultando da correlação entre os erros de medição do ERS e exposição. Ainda, é possível que a presença do ERS de interesse possa distorcer a percepção do participante com relação aos atributos físicos e sociais da UC (CAUGHY et al., 2001; DIEZ-ROUX, 2007).

Apesar de avanços na área, nota-se a necessidade de identificar características próprias das vizinhanças que são relevantes ao risco de um evento ou agravo à saúde, que contemplem as diversidades inter e intra-regionais. Faz-se necessário, portanto, o desenvolvimento de escalas que possibilitem comparaçóes regionais e 
que mensurem a vizinhança dentro de definições prévias dos eventos em saúde em estudo, com qualidades ecométricas e psicométricas, possibilitando posteriores análises dos fatores de risco nos vários níveis de observação.

\section{UC: observação social sistemática das características físicas e sociais}

Observação social sistemática pode ser definida como a observação direta das condições físicas e interações sociais que ocorrem na UC (RAUDENBUSH; SAMPSON, 1999). Utilizamos "observação social sistemática” (OSS) como tradução para o português da expressão em língua inglesa "systematic social observations".

Definida a UC de estudo, administrativa ou percebida, a observação social sistemática (OSS) apresenta-se como uma estratégia para a mensuração de seus atributos físicos e sociais. Segundo Sampson \& Raudenbush (1999), há aproximadamente 40 anos, Reiss sistematizou, no campo da sociologia, a OSS como estratégia para o estudo dos fenômenos sociais, detalhando suas vantagens, desvantagens e discutindo questôes metodológicas para sua utilização em outras áreas do conhecimento.

Atualmente, o que motiva a incorporação da OSS em estudos epidemiológicos é a existência de características físicas e sociais da vizinhança, consideradas variáveis integrais, que não podem ser capturadas de forma válida e confiável por meio de inquéritos populacionais. A OSS, portanto, pode ser utilizada para gerar medidas de atributos físicos e sociais da UC em vários níveis de agregação (ex. ruas, quarteirões, grupos de quarteirões, bairros) e indicadores tais como: estética do lugar (ex. presença ou ausência de lixos nas ruas, pichaçôes); territorialidade (ex. equipamentos de segurança, hostilidade); disponibilidade de áreas de lazer (ex. praças, jardins); facilitadores para o consumo de álcool e fumo (ex. presença ou ausência de bares e locais de venda de cigarros). Ainda, é possível medir interaçôes sociais que ocorrem no entorno físico da UC (ex. pessoas conversando e caminhando nas calçadas) (SAMPSON; RAUDENBUSH, 1999 e 2004; COHEN et al., 2000; CAUGHY et al., 2001; SCOTT et al., 2007; SUMINSKI et al., 2007).

O rigor metodológico necessário na condução da OSS não difere do utilizado em estudos epidemiológicos em geral, no que se refere à seleção da amostra, elaboração de instrumentos de coleta de informação e no controle e garantia da qualidade dos dados. Nesta metodologia, o observador tem papel similar ao do 
entrevistador, porém enquanto o entrevistador formula perguntas ao entrevistado, o observador busca as informações na observação da UC.

Entre as vantagens da OSS podemos citar a possibilidade de observar número considerável de diferentes atributos da UC e obtenção de informação independente e padronizada, não permeada pela percepção dos moradores, procurando reduzir o viés de memória e fonte comum de informação, dentre outros. No entanto, alguns construtos dependem do conhecimento e percepção dos moradores para sua mensuração, por exemplo, coesão social, variável integral não diretamente observável (DIEZ-ROUX, 2007).

Finalmente, a OSS vem sendo cada vez mais utilizada em saúde pública. Recentes estudos relatam a utilização desta metodologia para determinar a associação entre atributos físicos e sociais da UC e diversos ERS, comportamentos e estilos de vida. Podemos citar os estudos sobre atividade física (GAUVIN et al., 2005; SUMINSKI et al., 2006 e 2007), deterioração física estrutural (COHEN et al., 2000; CAUGHY et al., 2001) e urbanização (OLIVEIRA et al., 2006).

\section{Considerações finais}

A combinação de informações de múltiplas fontes, como dados administrativos, a vizinhança percebida, inquéritos populacionais e OSS, é relativamente recente no campo da saúde coletiva, e ainda necessita de maiores elaboraçôes conceitual, metodológica e analítica. Entretanto, o crescente interesse na área remete para a consolidação do marco teórico, onde a incorporação dos atributos físicos e sociais da UC às características individuais e agregadas daqueles ali aninhados compõe níveis hierárquicos de complexidade relevantes para o entendimento da ocorrência dos ERS nas populaçóes.

\section{Referências}

AUCHINCLOSS, A.H. et al. Association of insulin resistance with distance to wealthy areas: the multi-ethnic study of atherosclerosis. Am. J. Epidemiol., Baltimore, v.165, n.4, p. 389-397, 2007.

BALFOUR, J.L.; KAPLAN, G.A. Neighborhood environment and loss of physical activity in older adults: evidence from the alameda county study. Am. J. Epidemiol., Baltimore, v.155, n.6, p. 507-515, 2002.

BARATA, R.B. Epidemiologia social. Rev Bras Epidemiol., São Paulo, v.8, n. 1, p. 7-17, 2005.

BILD, D.E. et al. Multi-ethnic study of atherosclerosis: objectives and design. Am. J. Epidemiol., Baltimore, v.156, n.9, p.871-881, 2002. 
BLAKELY, T.A.; WOODWARD, A.J. Ecological effects in multi-level studies. J. Epidemiol. Community Health, London, v. 54, n.5, p. 367-374, 2000.

BOSLAUGH, S.E. et al. Perceptions of neighborhood environment for physical activity: is it "Who you are" or "Where you live"? J. Urban Health, New York, v.81, n.4, p. 671-681, 2004.

BOSMA, H.; VAN DE MHEEN, H.D.; BORSBOON, G.J. Neighborhood socioeconomic status and all cause mortality. Am. J. Epidemiol., Baltimore, v.153, n.4, p.363-371, 2001.

BRENNAN, L.K. et al. Linking perceptions of the community to behavior: are protective social factors associated with physical activity? Health. Educ. Behav., Califórnia, v.30, n.6, p.7 740-55, 2003.

BROWNSON, R.C. et al. Measuring the environment for friendliness toward physical activity: a comparison of the reliability of 3 questionnaires. Am. J. Public Health., New York, v.94, n.3, p. 473-483, 2004.

CAIAFFA, W.T. et al. Saúde urbana: "a cidade é uma estranha senhora, que hoje sorri e amanhã te devora”. Cien. Saude Col., Rio de Janeiro (no prelo).

The urban environment from the health perspective: the case of Belo Horizonte, Minas Gerais, Brazil. Cad. Saúde Pública, Rio de Janeiro, v. 21, n. 3, p. 958-967, 2005.

CAUGHY, M.O.; O'CAMPO P. J.; PATTERSON, J. A brief observational measure for urban neighborhoods. Health Place, Oxford, v.7, n. 3, p. 225-236, 2001.

CAUGHY, M.O. A brief observational measure for urban neighborhoods. Health Place, Oxford, v.7, n. 3, p. 225-236, 2001.

CHO, Y., PARK, G.S., ECHEVARRIA-CRUZ, S. Perceived neighborhood characteristics and the health of adult Koreans. Soc. Sci. Med., Oxford, v.60, n.6, p. 1285-1297, 2005.

CHO, Y.; PARK, G.S.; ECHEVARRIA-CRUZ, S. Perceived neighborhood characteristics and the health of adult Koreans. Soc. Sci. Med., Oxford, v.60, n.6, p. 1285-1297, 2005.

COHEN, D. et al. "Broken windows" and the risk of gonorrhea. Am. J. Public Health., New York, v. 90, n. 2, p. 230-236, 2000.

MATTOS ALMEIDA, M.C. de et al. Spatial vulnerability to dengue in a Brazilian urban area during a 7-year surveillance. J. Urban Health, New York, v.84, n.3, p.334-345, 2007.

DIEZ ROUX, A.V. et al. Availability of recreational resources and physical activity in adults. Am. J. Public Health, New York, v.97, n.3, p. 493-499, 2007.

DIEZ ROUX, A.V. Bringing context back into epidemiology: variables and fallacies in multilevel analysis. Am. J. Public Health, New York, v. 88, n.2, p. 216-222, 1998.

. Investigating neighborhood and area effects on health. Am. J. Public Health., New York, v.91, n.11, p.1783-1789, 2001.

. Residential environments and cardiovascular risk. J. Urban Health., New York, v.80, n.4, p.569-589, 2003. 
. The study of group-level factors in epidemiology: rethinking variables, study designs, and analytical approaches. Epidemiol. Rev., Baltimore, v.26, n.1, p.104-111, 2004.

DIEZ ROUX, A.V.; MERKIN, S.S.; ARNETT, D. Neighborhood of residence and incidence of coronary heart disease. N. Engl. J. Med., Massachusetts, v. 345, n.2, p. 99-106, 2001.

DIEZ ROUX, A.V.; NIETO, F.; MUNTANER, C. Neighborhood environments and coronary heart disease: a multilevel analysis. Am. J. Epidemiol., Baltimore, v.146, n.1, p.48-63, 1997.

DIEZ ROUX, A.V. Neighborhoods and health: where are we and where do we go from here? Rev. Epidemiol. Sante Publique, France, v. 55, n. 1, p. 13-21, 2007.

GAUVIN, L.; RICHARD, L. ; CRAIG, C.L. et al. From walkability to active living potential: an "ecometric" validation study. Am. J. Prev. Med., Amsterdan, v. 28, n. 2, p.126-133, 2005. Suplemento 2.

HARBURG, E.; ERFURT, J.; CHAPE, C. Socioecological stressor areas and black white blood pressure: Detroit. Journal of Chronic Disease, Oxford, v.26, n.9, p.585-611, 1973.

HARPHAM T.; GRANT, E.; THOMAS, E. Measuring social capital within health surveys: key inssue. Health Policy Plan., Oxford, v. 17, n. 1, p. 106-111, 2002.

JONES, K.; DUNCAN, C. Individuals and their ecologies: analysing the geography of chronical illnes within a multilevel modelling framework. Health Place, Oxford, v.1, n.1 , p.27-40, 1995.

KAWACHI, I.; BERKMAN, L.F. Introduction. In: . KAWACHI I.; BERKMAN L.F. Neighborhoods and health. New York: Oxford University Press, 2003. p. 1 - 19.

KRIEGER, N. Epidemiology and the web of causation. Has anyone seen the spider? Soci. Sci. Med., Oxford, v. 39, n.7, p.887-903, 1994.

LIMA-COSTA, M.F.F. A saúde dos adultos na Regiāo Metropolitana de Belo Horizonte: um estudo epidemiológico de base populacional. Belo Horizonte: Núcleo de Estudos em Saúde Pública e Envelhecimento, 2004. 132p.

MOORE, L.V.; DIEZ ROUX, A.V. Associations of neighborhood characteristics with the location and type of food stores. Am. J. Public Health, New York, v.96, n. 2, p. 325-331, 2006.

MOORE, L.V.; DIEZ ROUX, A.V.; BRINES, S. Comparing perception-based and geographic information system (GIS)-based characterizations of the local food environment. Journal of Urban Health., New York, v.85, n.2, p.206-216, 2008.

MORGAN, M.; CHINN, S. ACORN group, social class, and child health. Am J. Epidemiol., Baltimore, v.37, n.3, p.196-203, 1983.

MORLAND, K.; WING, S.; DIEZ ROUX, A.V. The contextual effect of the local food environment on resident's diets: the atherosclerosis risk in communities study. Am. J. Public Health. New York, v.92, n.11, p.1761-1767, 2002.

MUJAHID, M.S. et al. Assessing the measurement properties of neighborhood scales: from 
psychometrics to ecometrics. Am. J. Epidemiol., Baltimore, v.165, n.8, p. 858-867, 2007.

NIELSEN, T.S.; HANSEN, K.B. Do green areas affect health? Results from a Danish survey on the use of green areas and health indicators. Health Place, Oxford, v.13, n.4, p. 839-850, 2007. O'CAMPO, P.; GIELEN, A.; FADEN, R. Violence by male partners against women during the childbearing year: a contextual analysis. Am. J. Public Health., New York, v.85, n.8 p. 1, p.10771082, 1995.

OLIVEIRA, C.D.L. et al. A case-control study of microenvironmental risk factors for urban visceral leishmaniasis in a large city in Brazil, 1999 - 2000. Rev. Panam. Salud Publica, Washington, v.20, n.6, p. $369-376,2006$.

OMPAD, D.C. et.al. Social determinants of the health of urban populations: methodologic considerations. J. Urban Health., New York, v.84, n.1, p. 42-53, 2007.

PICKETT, K.E., PEARL, M. Multilevel analyses of neighbourhood socioeconomic context and health outcomes: a critical review. J. Epidemiol. Community Health, London, v. 55, n.2, p.111122, 2001.

PICKETT, K.E.; PEARL, M. Multilevel analyses of neighbourhood socioeconomic context and health outcomes: a critical review. J. Epidemiol. Community Health, London, v. 55, n.2, p.111-122, 2001.

RAUDENBUSH SW. The quantitative assessment of neighborhood social environments. In: . KAWASHI Ichiro; BERKMAN Lisa F. (Ed.). Neighborhoods and health. New York: Oxford University Press, 2003. p.112-131.

RAUDENBUSH, S.W.; SAMPSON, R.J. Ecometrics: toward a science of assessing ecological settings, with application to the systematic social observation of neighborhoods. Sociologic Methodology, University Michigan, v.29, p.1 - 41, 1999.

REJNEVEL, S. The impact of individual and area characteristics on urban scocioeconomic differences in health and smoking. Int. J. Epidemiol., Oxford, v.27, n.1, p.33-40, 1998.

SAMPSON, R.J.;MORENOFF, J.D.; GANNON-ROWLEY, T. Assessing "neighborhood effects": social processes and new directions in research. Annu. Rev. Sociol., Califórnia, v. 28, p. 443-78, 2002.

SAMPSON, R.J.; RAUDENBUSH, S.W. Seeing Disorder: neighborhood stigma and the social construction of "broken windows". Soc. Psychol. Q., Ohio, v. 67, n. 4, p. 319-342, 2004.

SAMPSON, R.J.; RAUDENBUSH, S.W. Systematic social observation of public spaces: a new look at disorder in urban neighborhoods. AJS, Chicago, v. 105, n. 3, p.603-51, 1999.

SANTOS, S.M. et al. Associação entre fatores contextuais e auto-avaliação de saúde: uma revisão sistemática de estudos multinível. Cad. Saúde Pública, Rio de Janeiro, v. 23, n. 11, p. 2533 - 2554, 2007. 
SCHWARTZ, S.; SUSSER, E.; SUSSER, M. A future for epidemiology? Annu Rev Public Health,

Califórnia, v.20, p.15-33, 1999.

SCOTT, M.M., EVENSON, K.R., COHEN, D.A. et al. Comparing perceived and objectively measured access to recreational facilities as predictors of physical activity in adolescent girls. $J$. Urban Health, New York, v. 84, n. 3, p. 346-59, 2007.

SILVA, M.J. et al. Psychometric and cognitive validation of a social capital measurement tool in Peru and Vietnam. Soc. Sci. Med., Oxford, v. 62, n.4, p. 941-953, 2006.

SLOGGETT, A.; JOSHI, H. Deprivation indicators as predictors of life events 1981 - 1992 based on the UK ONSS longitudinal study. J. Epidemiol Community Health., London, v.52, n.4, p.228-233, 1998.

SUMINSKI, R.R. et al. Characteristics of urban sidewalks/streets and objectively measured physical activity. J. Urban Health, New York, v. 85, n. 2, 2007.

SUMINSKI, R.R.; PETOSA, R.L; STEVENS, E. A method for observing physical activity on residential sidewalks and streets. J. Urban Health, New York, v. 83, n. 3, p. 434-443, 2006.

SUSSER M.; SUSSER, E. Choosing a future for epidemiology: II. From black boxes to chinese boxes and eco-epidemiology. Am J. Public Health., New York, v. 86, n. 5, p.674-677, 1996.

. Choosing a future for epidemiology: I. Eras and paradigms. Am J. Public Health., New York, v.86, n.5, p.668-673, 1996.

VLAHOV, D. et al. Urban as a determinant of Health. J. Urban Health., New York, v.84, n.1, p16-26, 2007.

WALKER, R.B.; HILLER, J.E. Places and health: a qualitative study to explore how older women living alone perceive the social and physical dimensions of their neighbourhoods. Soc. Sci. Med., United Kingdom, v.65, n.6, p. 1154-1165, 2007.

WERNECK, G.L. et al. Multilevel modelling of the incidence of visceral leishmaniasis in Teresinha, Brazil. Epidemiol. Infect. Cambridge, v.135, n.2, p. 195-201, 2007.

YANG, M.J. et al. Development and validation of an instrument to measure perceived neighbourhood quality in Taiwan. J. Epidemiol. Community Health, London, v.56, n.7, p. 492 496, 2002. 


\section{Abstract}

\section{Context unit and systematic social observation: a review of concepts and methods}

We understand that "where one lives makes a difference to health in addition to who you are", and that the effects of the place of residence or context unit (CU) on public health are due to the heterogeneity of the physical and social environment characteristics, in addition to the individual and aggregate attributes of the population nested in the CU. Those attributes, although intrinsically dependent on the individuals, are typically external to them and susceptible to intervention. Also, the UC's are understood as ecological units nested within successively larger communities. Depending on the study hypothesis, census-defined areas may be used as proxy for the CU. Alternatively, the CU may be defined by the individual's perception of his/her neighborhood. The renewed interest on the health effects of the CU are associated with new trends in public health, namely: new hierarchical levels of exposure beyond individual level characteristics, inequalities and social determinants of health, urbanization and the need to evaluate interventions not traditionally associated to public health. Our objective was, first, to review options while choosing the relevant $\mathrm{CU}$ and second, to review strategies to determine and quantify the characteristics of the $\mathrm{CU}$ using social systematic observation (SSO). The combination of census-defined data, information on the neighborhood defined by the local population, surveys and SSO still needs conceptual, methodological and analytical development. However, the distribution of the physical and social attributes of the CU will permit to incorporate other hierarchical level of complexity to better understand the incidence and prevalence of health related events in populations.

> Key words: urban health; neighborhood; systematic social observation. 